\title{
Cells, circuits, and choices: Social influences on perceptual decision making
}

\author{
Andreas MojZisch \\ Georg-August-Universität, Göttingen, Germany \\ AND \\ KRISTINe KRUG \\ University of Oxford, Oxford, England
}

\begin{abstract}
Making decisions is an integral part of everyday life. Social psychologists have demonstrated in many studies that humans' decisions are frequently and strongly influenced by the opinions of others - even in simple perceptual decisions, where, for example, participants have to judge what an image looks like. However, because the effect of other people's opinions on decision making has remained largely unaddressed by the neuroimaging and neurophysiology literature, we are only beginning to understand how social influence is integrated into the decision-making process. We put forward the thesis that by probing the neurophysiology of social influence with perceptual decisionmaking tasks similar to those used in the seminal work of Asch $(1952,1956)$, this gap could be remedied. Perceptual paradigms are already widely used to probe neuronal mechanisms of decision making in nonhuman primates. There is also increasing evidence about how nonhuman primates' behavior is influenced by observing conspecifics. The high spatial and temporal resolution of neurophysiological recordings in awake monkeys could provide insight into where and how social influence modulates decision making, and thus should enable us to develop detailed functional models of the neural mechanisms that support the integration of social influence into the decision-making process.
\end{abstract}

An experienced dermatologist has to decide whether a mole on the sole of a patient's foot looks suspicious for melanoma. After she examines it carefully, she thinks that the mole is benign. Then she is told that two other dermatologists have diagnosed melanoma and said that the mole should be removed. What should the dermatologist do? Now, consider a monkey foraging for food in the trees. The fruit on its current tree has turned out not to be very juicy, so the monkey moves on. In three directions, it sees a few spots of color glinting between the leaves that might indicate fruit; it remembers vaguely that some of these might belong to a tree that yielded nice fruit only yesterday, and it sees another monkey moving about in one of the trees. Which tree should the first monkey move to next?

Obviously, both dermatologists and monkeys are faced with decisions every day of their lives. Often, as illustrated by the examples above, decisions are made in a social context. Thus, the decision of the dermatologist is likely to be influenced by hearing what her colleagues think (Bonaccio \& Dalal, 2006; Yaniv, 2004). Similarly, observing a conspecific foraging in a tree nearby may be a cue for a monkey, biasing its decision about where to move next (Bonnie \& de Waal, 2007; Meunier, Monfardini, \& Boussaoud, 2007; Myers, 1970; Subiaul, Cantlon, Holloway, \& Terrace, 2004).

In general, three aspects of social decision making can be distinguished: The first aspect refers to situations in which the success of one's own decision directly depends on the concomitant decisions of others. To illustrate, imagine deciding about the level of a first request in a negotiation about a pay raise. The level at which the first request is pitched will, on the one hand, be dependent on its presumed reception by the other person; on the other hand, it also has direct consequences for the offer given in response. In most of the scenarios used for research on such social interactions, the decision maker is in direct competition with others for a specific reward. We will refer to this aspect of social decision making as strategic decision making. Utilizing tasks derived from game theory (von Neumann \& Morgenstern, 1944), neuroeconomics has recently begun to successfully delineate the neuronal circuits that contribute to strategic social decision making (for reviews, see Fehr \& Camerer, 2007; Glimcher, 2003; Loewenstein, Rick, \& Cohen, 2008; Sanfey, 2007). Neuroimaging experiments and brain lesions in humans and animals have identified an important role in strategic decision making for brain areas that are involved in reward evaluation, reinforcement learning, and the representation of mental states of others, such as the ventral striatum, caudate nucleus, orbitofrontal cortex, insula, and anterior cingulate cortex.

The second aspect of social decision making is group decision making. In contrast to strategic decision making, group decision making requires a number of decision makers to cooperate and agree on one of several choice options. Group decision making has been examined extensively by

A. Mojzisch, mojzisch@psych.uni-goettingen.de 
social psychologists (for reviews, see Hastie \& Kameda, 2005; Kerr \& Tindale, 2004). Whereas early research focused on the rules that govern moving from a diverse set of individual preferences to agreement on a consensus decision, more recent work has examined how group members pool and integrate their diverse expertise and knowledge (e.g., Mojzisch, Schulz-Hardt, Kerschreiter, Brodbeck, \& Frey, 2008; Schulz-Hardt, Brodbeck, Mojzisch, Kerschreiter, \& Frey, 2006). So far, however, the neuronal correlates of the processes involved have not been examined because of the complex nature of group decision making.

The third aspect of social decision making refers to situations in which the decision is in the hands of a single person, but the decision maker is also subject to the influence of the opinions of other people. This form of social influence affects decision making in a wide range of circumstances, such as medical, economic, or political decision making (e.g., De Dreu \& Beersma, 2001; Larson, Christensen, Abbott, \& Franz, 1996; Levine \& Kaarbo, 2001). For example, when politicians make decisions about how to reform a particular set of laws, they often consult the opinions of experts in the field. Similarly, a woman buying a dress is likely to be influenced by the opinions of peers. There is a long research tradition in social psychology on the influence of the opinions of others on decision making, yet the cognitive mechanisms through which they affect decision making are still a matter of intense debate (Asch, 1952; Sherif, 1936; for recent reviews, see Cialdini \& Goldstein, 2004; Martin \& Hewstone, 2003). At the same time, social influence on decisions is only beginning to be addressed by neuroscience.

In this article, we focus on the latter aspect of social decision making, in particular on studies that probe social influence on perceptual decisions. We argue that through studying the effects of social influence on perceptual decision making, we can potentially probe deeper into the brain mechanisms through which social factors exert their influence. There are many studies in which social psychologists have used perceptual paradigms to examine the influence of other people's opinions on decision-making processes (e.g., Asch, 1952, 1956; Martin, 1998; Moscovici, Lage, \& Naffrechoux, 1969; Moscovici \& Personnaz, 1980). Similarly, neurophysiologists have utilized perceptual paradigms to unravel the neuronal circuitry underlying perceptual decision making in the monkey (e.g., Britten, Newsome, Shadlen, Celebrini, \& Movshon, 1996; Dodd, Krug, Cumming, \& Parker, 2001; Newsome, Britten, \& Movshon, 1989; Shadlen \& Newsome, 1996; Uka $\&$ DeAngelis, 2004). It is therefore surprising that there are so few data on the neuronal correlates of social influence on decision making. Decision making in humans is clearly more complex than it is in monkeys. However, as we will show, there is evidence that monkeys' behavior is affected by the behavior of their conspecifics in a way that allows the adaptation of paradigms of social influence on perceptual decision making from social psychology for use with rhesus monkeys. Through neurophysiological recordings in awake behaving monkeys, we can probe directly, at the level of single neurons, the mechanisms by which social influence affects the neural computation of decisions. Thus, we can not only examine the levels of brain computation at which the integration of social influence into the decision process might take place, but also how social influence might be integrated with sensory evidence and/or decision signals.

First, we review evidence from social psychology demonstrating the tremendous impact of social influence on perceptual decisions. In the second section, we explore recent neuroimaging work in humans on the neural correlates of social influence in such decision tasks. In the third section, we first review evidence for social influence on monkey behavior. Then, we turn to neurophysiological studies on perceptual decision making in the monkey, where we briefly lay out how information from different sources might be integrated in order for the brain to compute perceptual decisions. Finally, we describe and discuss a potential experiment that aims to identify the neuronal mechanisms underlying the effect of social influence on perceptual decision making.

\section{Evidence From Social Psychology: The Effects of Other People's Opinions on Perceptual Decisions}

Take a look at Figure 1. Compare the line on the left side with each of the three lines $(\mathrm{A}, \mathrm{B}, \mathrm{C})$ on the right. Which of the three lines on the right is the same length as the single line on the left?

Okay, that was easy: It is obviously C. Now, imagine you are sitting in a lab with 6 other participants in a study on perceptual decision making. Again, you have to decide which of the three lines on the right has the same length as the line on the left. You and the other participants take turns, in a fixed order, calling out the answer. Imagine that you are the 6th after 5 other participants have unanimously answered "A." Would you still say that the answer is C?

A similar experiment was conducted by the renowned psychologist Solomon Asch in the early 1950s (Asch, 1952, 1956). He set out to prove that when people are faced with making a decision about an unambiguous stimulus, other people's choices should have no effect (at least that was what he thought). Participants were shown three lines, with another line for comparison, similar to the diagram
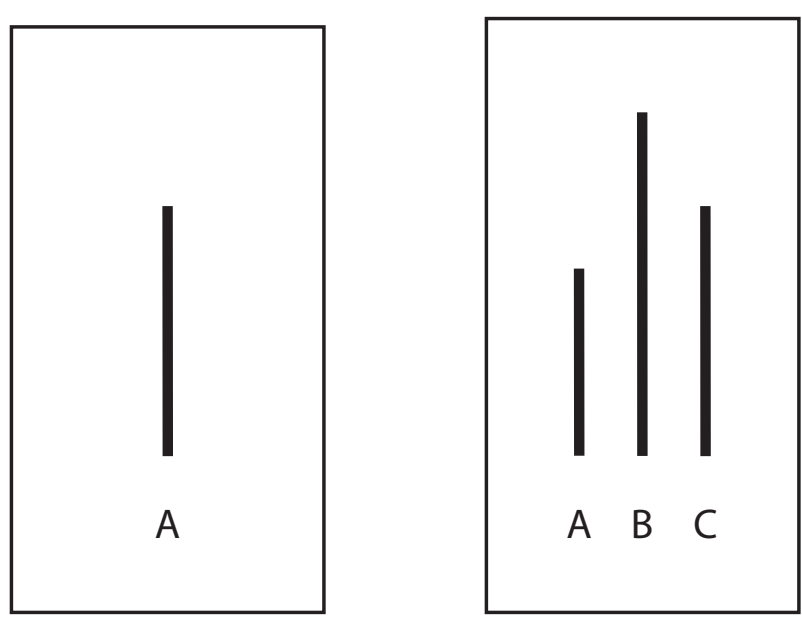

Figure 1. An example of the stimuli used in the Asch (1952, 1956) experiments. 
in Figure 1. What participants were not aware of was that they were the only "real" participant in the room; all the other people were confederates of the experimenter, who had been instructed to unanimously give a predetermined answer. Each participant took part in 18 trials, using variants of the stimulus. On 6 trials, the confederates were instructed to give correct answers; on the other 12 trials, they unanimously answered incorrectly. The "real" participant, who knew nothing of this, had to answer second to last.

The results were intriguing and not at all what Asch $(1952,1956)$ had predicted. On average, participants responded with the group, giving a wrong answer on $37 \%$ of the trials. Not every participant gave so many incorrect answers, but $76 \%$ of the participants conformed at least once with an obviously wrong answer, and $27 \%$ yielded to group pressure on two thirds or more of the trials. In contrast, in a control condition, in which participants made their decisions in isolation, the error rate was a negligible $0.7 \%$. The results reveal the enormous impact of an obviously incorrect majority on perceptual decision making.

One might argue that participants were agreeing with the majority only because of the public nature of the required response. In one of the Asch experiments (Asch, 1956, Experiment 4), participants were made to believe that they had arrived late, and that they were therefore to write down their responses privately. As expected, the rate of conformity decreased significantly, thereby showing that less exposure to group pressure reduces conformity. However, participants still conformed to wrong answers by the confederates on $12.5 \%$ of all critical trials, which was significantly higher than the error rate in the control condition $(0.7 \%)$.

Two main reasons have been identified for why people tend to go along with a majority (Deutsch \& Gerard, 1955): First, people have a need for acceptance and social approval. Conformity caused by the desire to be liked and accepted has been termed "normative influence." Normative influence comes into play if disagreeing with others may lead to social rejection. Second, people have a need to feel confident that their perceptions and attitudes are correct. Conformity caused by the desire to determine what is right has been termed "informational influence." Informational influence comes into play if we are motivated to be accurate and if we are uncertain, for example, because the stimulus is ambiguous. Normative influence was likely to be the main cause of conformity in the Asch $(1952,1956)$ studies because stimuli were unambiguous, thus reducing the likelihood that informational influence was operating. Furthermore, participants were under surveillance by the majority, increasing the likelihood that normative influence was operating. However, normative influence was probably not the exclusive cause of conformity, because conformity was not completely eliminated if participants were allowed to write down their answers privately.

After his experiments, Asch $(1952,1956)$ asked his participants why they went along with the majority. Many participants reported that they had been aware that they saw things differently than the others, but felt that they might have been inaccurate and that the majority was correctimplying informational influence on their judgment. Other participants were not convinced that the majority was cor- rect but conformed because they were afraid of being the lone dissenter. These comments suggest a normative influence of the incorrect majority on the response the participant gave. However, others reported that they had actually seen the lines the way the majority reported. In other words, the answers of these participants suggest that the opinions of others might influence perceptual decision making through an actual change in perception. How social influence affects decision making remains unclear.

Another important question is how much bearing Asch's $(1952,1956)$ results have on perceptual decisions we potentially face in everyday life. Note that Asch's participants had nothing to lose if they went with the majority, because giving the correct answer was not of great importance to them. Hence, it may have been easier to go along with the majority. In contrast, in real life, our decisions often have important consequences, and we want to get things right.

Baron, Vandello, and Brunsman (1996) employed a decision-making task that was closer to real life than the task used by Asch $(1952,1956)$ : eyewitness identification. Using a paradigm similar to that in the Asch studies, the task took place in a group, consisting of 1 participant and 3 confederates. On each trial, participants first saw a slide showing a "perpetrator." Next, they were shown a slide of a lineup consisting of four men, one of whom was the perpetrator; they were asked to pick out the perpetrator. The main aim of the study was to examine the effect of task importance on social influence. In the high-importance condition, participants were told that the task was a real eyewitness identification test that would be employed by courts to assess eyewitnesses' identification ability. Participants' scores would be used to establish a norm for performance. Moreover, they were told that those who scored the highest would win \$20. In the low-importance condition, participants were told that the task was still in a state of development; and no monetary incentives for accuracy were provided. Baron et al. also manipulated task difficulty. In the low-difficulty condition, each slide was presented for $5 \mathrm{sec}$, whereas in the high-difficulty condition, each slide was presented for $0.5 \mathrm{sec}$.

The results revealed that participants in the low-difficulty, low-importance condition (i.e., the condition that was similar to the setting in Asch, 1952, 1956) went along with an incorrect majority on $33 \%$ of the critical trials (a result very similar to those of Asch, 1952, 1956). In the low-difficulty, high-importance condition, the conformity rate dropped, but participants still conformed on $16 \%$ of the trials. In the corresponding control condition without social influence, participants made mistakes on only $3 \%$ of the trials. Thus, in the case of an easy decision-making task, increasing task importance reduced but did not eliminate conformity. A different pattern emerged in the high-difficulty conditions. Participants in the high-difficulty, low-importance condition went along with the majority on $35 \%$ of the trials, similar to the results of the low-difficulty condition. In contrast, in the high-difficulty, high-importance condition, conformity increased to $51 \%$. Thus, if the task is difficult, being motivated to be accurate increases the effect of social influence. In sum, the results show that social influence also has an effect on perceptual decision making if real-life tasks 
are used, and that increasing task importance may even increase the impact of social influence if the task is difficult.

So far, we have seen examples of the tremendous impact of majority influence on perceptual decision making. But can a majority also be influenced by a disagreeing minority? The French social psychologist Serge Moscovici (Moscovici, 1976, 1980) proposed that minorities can be successful in exerting influence on majorities, depending on the behavioral style the minority adopts. By using a reversed Asch paradigm, Moscovici et al. (1969) showed 4 naive participants and 2 confederates a series of unambiguously blue slides that varied only in their light intensity. When the confederates (a numerical minority) called the blue slides "green" on every trial, the participants also called the blue slides "green" on $8.45 \%$ of the trials, which was significantly different from the control condition with no confederates $(0.25 \%)$. But when the confederates were inconsistent, minority influence dropped to nonsignificant levels. More generally, the idea that minority factions can have a profound influence on majority members has been found not only in simple perceptual decision-making tasks, but also in more complex decision processes, such as political or organizational decision making (for reviews, see De Dreu \& Beersma, 2001; Levine \& Kaarbo, 2001).

Following on from their earlier work, Moscovici and Personnaz (1980) proposed that a minority may exert its effect by altering the underlying perceptual processes themselves, whereas majority influence yields only verbal compliance. Participants were shown a series of blue slides to which they had to make two responses: (1) Name the color of the slide and (2) make an afterimage judgment of the slide. An afterimage is what one sees when viewing a white screen after having viewed a colored screen for a certain period. In particular, it is the complementary color of the stimulus (the afterimage of blue is yellow, and the afterimage of green is red-purple). Remarkably, the results turned out as predicted by conversion theory (Moscovici, 1980): If participants were exposed to a dissenting majority, the afterimage was yellow, consistent with having seen a blue slide. However, if participants were exposed to a dissenting minority, the afterimage shifted toward purple, indicating that participants had indeed seen a green slide. These results suggest that minority influence is able to produce a genuine change in perception. However, the results are controversial, and other studies failed to replicate these findings (e.g., Doms \& Van Avermaet, 1980; Martin, 1998; Sorrentino, King, \& Leo, 1980).

In summary, mounting evidence from social psychology suggests that perceptual decision making is influenced by other people's decisions. Even if a majority is blatantly wrong and even if group pressure is reduced to a minimum, social influence works. The effect of social influence initially demonstrated in studies on perceptual decisions has also been found in areas of more complex decision processes, such as, for example, medical, political, and organizational decision making. Social psychologists have suggested possible mechanisms through which social influence affects decision making: normative and informational influence. There is some indication that social influence cannot only affect the response conveying the decision, but may, at least in some circumstances, have a direct influence on the perceptual processes that contribute to the decision. However, we still do not know at which levels of the decision process social influence is integrated (i.e., lower level sensory vs. higher order decision-related processes). Debates between social psychologists usually revolve around psychological constructs, such as compliance versus conversion. In contrast, cognitive neuroscience allows us to open the black box of the mind and directly observe the brain at work. Hence, in what follows, we focus on research aiming to identify the neural correlates of social influence in both humans and monkeys.

\section{Neural Correlates of Social Influence on Decision Making in Humans}

One way to study how social information exerts its influence is by identifying the level of brain processing at which corresponding signals can be found. Unfortunately, the question of which brain mechanisms are at work when we integrate others' opinions during decision making has largely escaped the attention of neuroscientists. To the best of our knowledge, only one fMRI study has examined the neural correlates of social influence during decision making (Berns et al., 2005). Instead of having participants decide between different line lengths, as in Asch $(1952,1956)$, Berns et al. asked participants to mentally rotate 2-D line drawings of 3-D objects to decide whether two objects were the "same" or "different" (Shepard \& Metzler, 1971). Mental rotation tasks have been found to be associated with increased brain activity in the intraparietal sulcus (e.g., Jordan, Heinze, Lutz, Kanowski, \& Jäncke, 2001). The participants were told that others would be doing the same task, but that they were the only ones who would be in the scanner. The answers of 4 other "participants" were displayed on a computer screen, before the naive participant gave his or her answer while being scanned. The displayed answers were unanimously wrong in some instances and unanimously correct in others. Trials with mixed answers were randomly interspersed to make the experiment more believable, but these trials were not included in the analysis.

On average, participants agreed with the unanimous majority on wrong answers $41 \%$ of the time. Importantly, the brain activity of those who went along with the majority was markedly different from those who took independent positions. When people concurred with wrong answers, activity increased in the intraparietal sulcus and in the superior occipital gyrus. Put differently, going along with the group increased activity particularly in the neural network that normally is involved in mental rotation. Berns et al. (2005) concluded from these results that participants seemed to see the wrong answer under social pressure. In contrast, participants who did not conform with the others showed increased amygdala and caudate activity, possibly signifying an emotional toll for standing up for one's belief.

It is important to note that the task used by Berns et al. (2005) was considerably more difficult than the task used by Asch $(1952,1956)$, in which the stimuli were unambiguous. Indeed, the error rate in the control condition was $13.8 \%$ in the Berns et al. study, but only $0.7 \%$ in the Asch study. Therefore, it is conceivable that participants of Berns 
et al. were more uncertain whether their decision was right or wrong than were participants in the Asch study. Hence, it is likely that conformity in the Berns et al. study was primarily caused by the desire to reduce uncertainty (i.e., informational influence). However, normative influence may also have played a role, because the error rate was significantly higher when the group gave the incorrect information $(41 \%)$ than when the incorrect information was identified as being generated by the computer (32\%).

One caveat of the Berns et al. (2005) study is that the brain activity observed in those trials in which participants conformed may not have been causally involved in the participants' decision to conform. Participants may have been more likely to conform on trials on which they were uncertain about what the correct answer was. Hence, the increased activity may have been the result of participants paying extra attention to the stimuli. Similarly, participants who made more mental rotations to account for the differences between their perception and the group's judgment might have been more likely to conform.

Finally, and perhaps most importantly, it would be premature to interpret the increased activity in the parietal cortex observed by Berns et al. (2005) as evidence for the involvement of perceptual processes during conformity. Whereas the superior occipital gyrus is generally made up of visual areas, the parietal cortex links sensory to motor areas. As a consequence, neuronal activity in the parietal cortex is neither strictly sensory nor motor. With regard to perceptual decision tasks, neurons in the parietal cortex can represent the process of evidence integration for decision making as well as the decision itself (for reviews, see Gold \& Shadlen, 2007; Heekeren, Marrett, \& Ungerleider, 2008). Therefore, the activity in the parietal cortex observed by Berns et al. may not necessarily reflect a sensory processing change but may be related to the decision itself. In other words, if the parietal cortex serves to integrate all decision-relevant information, then the effects of social influence should be observed here, irrespective of whether other people's opinions affect perceptual or decision processes.

The idea that the parietal cortex may be more generally involved in integrating others' opinions during decision making is supported by a neuropsychological study on advice utilization (Gomez-Beldarrain, Harries, GarciaMonco, Ballus, \& Grafman, 2004). In this study, participants were asked to imagine being the director of a company, and to assess and integrate the recommendations of different advisors who forecast how well particular products would sell. The quality of the advice differed between the advisors. Participants were required to assess how accurate each of the advisors' forecasts was, and to make their own forecasts by combining the advisors' judgments. The results showed that patients with frontal lobe lesions performed significantly worse in advice utilization than did healthy control participants. In particular, patients with orbitofrontal lesions had severe problems in assessing advice quality. But more importantly for our discussion here, patients with parietal lobe lesions (who were included as a control group) showed no deficits in assessing advice quality but were impaired in integrating the advice according to its perceived quality. These findings underscore the idea that the parietal cortex may be important for integrating the opinions of others during decision making.

How do these results compare with the large literature investigating the neuronal correlates of strategic decision making? Whereas Berns et al. (2005) found increased activity in the occipital and parietal cortex, neuroeconomic research has predominantly implicated prefrontal areas and areas that are part of the brain's reward system - such as the ventral striatum and the caudate nucleus - as contributing to decisions when we compete and cooperate with others for rewards (Fehr \& Camerer, 2007; Loewenstein et al., 2008; Sanfey, 2007). The fact that the human reward system is implicated suggests that how we feel about other people's behavior might influence many of our decisions. The role of prefrontal cortex in social decision making is more controversial, but there is at least a growing consensus that the prefrontal cortex seems to be associated with processes by which more deliberative goals (such as the temptation to resist unfair offers) can be implemented (e.g., Knoch \& Fehr, 2007). Research from social neuroscience in humans has found that feeling socially rejected is correlated with neural activity in the anterior cingulate (e.g., Somerville, Heatherton, \& Kelley, 2006). This is supported by lesion experiments in the rhesus monkey, where the anterior cingulate has been associated with reduced interest in conspecifics (Rudebeck, Buckley, Walton, \& Rushworth, 2006; Rushworth, Behrens, Rudebeck, \& Walton, 2007). Hence, social influence caused by the desire to avoid social rejection (normative influence) may putatively be associated with activity in the anterior cingulate.

In sum, these studies provide a first step toward understanding the neural correlates of social influence during decision making. Frontal and reward circuits appear well placed to contribute to the deliberative and emotional evaluation of social influences, whereas the results by Berns et al. (2005) and Gomez-Beldarrain et al. (2004) suggest that information about others' opinions during decision making could be integrated in the parietal cortex and/or occipital cortex for perceptual tasks. More evidence is needed to delineate more precisely the role that the parietal and occipital cortex play in other perceptual decision tasks as well as under conditions of informational and normative social influence. However, whereas the results from the imaging studies provide us with an indication of which brain regions might be involved, they do not reveal their functional interactions. Thus, if we want to understand the functional mechanisms that underlie the impact of social influence on decision-making processes, we have to turn to the more precise spatial and temporal resolution of neurophysiological studies at the level of single neurons in the monkey. Single-cell recordings in the parietal cortex have already revealed the buildup of evidence for and against specific decisions until a decision is reached, as we describe below (see also Gold \& Shadlen, 2007).

\section{Decision Making in Monkeys: \\ Neural Correlates and Social Influence}

Evidence for social influence on decision making in monkeys. Remember the monkey in its tree foraging for food? The information about another monkey in a nearby 
tree could of course be interpreted as potential competition. However, it could also be taken simply as additional information about where good food might be found. If we want to study the neuronal processes underlying perceptual decision making using neurophysiology in the monkey, we need to show first that monkeys' behavior and decisions can be subject to social influences in a way similar to how humans' behavior and decisions are. Of course, monkeys cannot influence conspecifics by providing a verbal opinion, but they could influence others through their behavior.

At first glance, this might not be as straightforward for monkeys as one might imagine, because monkeys are generally thought to be poor imitators (Visalberghi \& Fragaszy, 2002). For instance, Zuberbühler, Gygax, Harley, and Kummer (1996) described a group of captive macaques, in which one male spontaneously learned how to retrieve apples with a stick from under a fence. Although other monkeys in the group started handling pieces of wood nearby, they produced little goal-directed imitation. Only months later did a small number of monkeys exhibit the same behavior with success. However, the animals' inappropriate handling of sticks gives some indication that what they saw their conspecifics do had probably affected their behavior. That monkeys' behavioral choices are affected by what they see is confirmed by capuchins' rejection of a lower reward when they see a conspecific receive a higher reward for the same task (Brosnan \& de Waal, 2003). Also, the foraging choices of conspecifics have been shown to influence other primates' choices to forage in a certain place (Bonnie \& de Waal, 2007). Bonnie and de Waal used a task in which whether rewards were available to observers and models varied across experimental conditions, such that rewards were available to either none, one, or both monkeys. Their results showed that capuchin monkeys do not need to be rewarded and do not even need to see the other monkey being rewarded to be influenced by it. It thus appears that primates find it intrinsically rewarding to observe and learn from their conspecifics (see also de Waal, 1998).

There is a growing literature describing observations of socially biased learning across different species of monkeys, though it appears to vary from species to species and also depends on the task (for a review, see Fragaszy \& Visalberghi, 2004). If we want to utilize this type of social behavior to study effects of social influence at the neuronal level, we ideally want to be able to show (1) that it is present in macaque monkeys (the main animal model in neurophysiological studies), (2) that macaques can learn arbitrary rules in this way, and (3) that this behavior can be reproduced reliably in the laboratory. Importantly, two studies of perceptual learning experiments have convincingly shown that rhesus monkeys can learn abstract rules by watching conspecifics (Myers, 1970; Subiaul et al., 2004). For example, Subiaul et al. trained 2 animals to respond in a specific order to sets of four photographs displayed on a touch screen (Figure 2). They learned sequences of new sets of photographs faster if they had been allowed to observe an expert monkey execute the same sequence. In observation and execution trials, the spatial configuration of the four photos was changed. Thus, the monkeys did not simply learn by motor imitation; rather, they learned the abstract rule of an arbitrary stimulus-response association. In a recent study, Meunier et al. (2007) confirmed that such behavior is indeed part of the natural repertoire for macaques and can be elicited without complex training paradigms. They showed that non-food-restricted rhesus monkeys can benefit from spontaneous observation of a conspecific to acquire new cognitive rules. They also confirmed the results of Subiaul et al. that performance improvements after observing a conspecific learning a new task cannot be accounted for by social facilitation (performance improvement resulting from the mere presence of a conspecific). In sum, monkeys' behavioral choices are influenced by the specific pattern of behavior of their conspecifics - in the wild as well as in the laboratory.

Single neurons and perceptual decision making. What paradigm could we use to study the neurophysiolog-

\section{A}

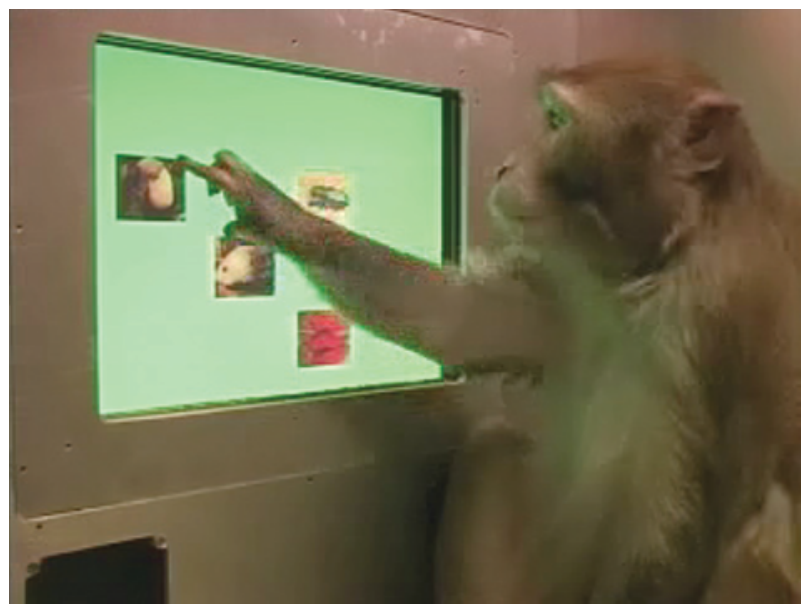

B

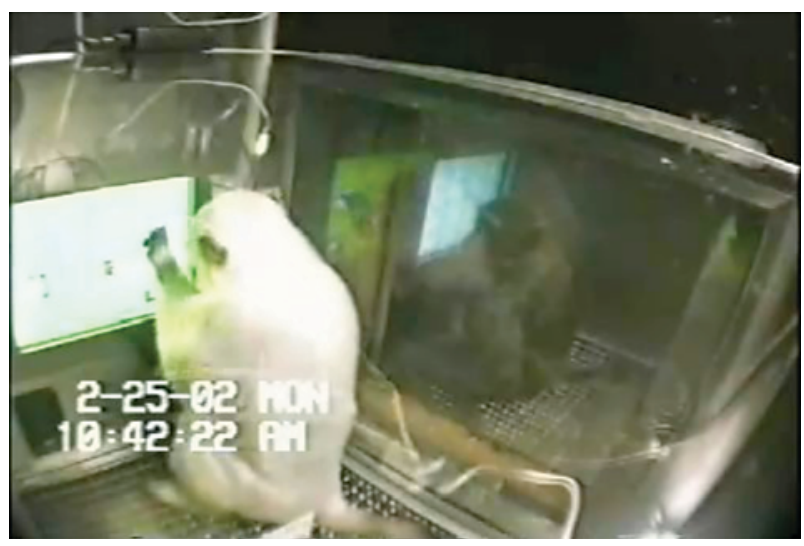

Figure 2. The experimental setting used by Subiaul et al. (2004). (A) A rhesus monkey carrying out the sequential task. Screen shot from subiaul.com/videos.html. Reprinted with permission from Herbert Terrace (Columbia University). (B) The expert monkey (left) is carrying out the task while the observer (right) watches through a screen. Adapted from Subiaul et al. (2004, supporting online material). Reprinted with permission from AAAS. 
ical correlates of social influence in perceptual decision making? Visual discrimination of motion and depth has been used widely to unravel the basic processes underlying perceptual decision making in the awake macaque (e.g., Britten et al., 1996; Dodd et al., 2001; Krug, Cumming, \& Parker, 2004; Newsome et al., 1989; Roitman \& Shadlen, 2002; Uka \& DeAngelis, 2004). The two major areas identified to contribute to these decision processes are extrastriate visual area V5/MT and the lateral intraparietal cortex (LIP), which are situated in the same general regions in which Berns et al. (2005) measured increased activation during social conformity in decision making.

One example of a perceptual decision task is the discrimination of direction of motion in a patch of random dots, which are moving with no overall motion vector (Newsome et al., 1989) (Figure 3). Such stimuli allow - at the level of single neurons - the distinction between representations of visual information driven by the stimulus itself and neuronal signals relating to decisions about the interpretation of that stimulus. Random dot patches showing coherent motion in a particular direction are used to determine neurons' response preferences and to train monkeys to report the perceived direction of motion via eye-movement responses. Using a two-alternative forced choice paradigm, Britten and colleagues (1996) have identified neurons in visual area V5/MT whose responses correlated trial by trial on a low but significant level with the decisions the monkey made about the direction of motion in a patch of randomly moving dots. This was true even when identical stimuli were used on each trial. Thus, neuronal correlates of perceptual evidence for a decision can be found in parts of extrastriate visual cortex. Similarly, perceptual evidence for visual depth is also represented in area V5/MT (Dodd et al., 2001; Uka \& DeAngelis, 2004). But we are not just dealing with correlates here. Electrically stimulating neurons in V5/MT influence the perceptual decisions monkeys make in accordance with the direction (or depth) preference of the stimulated neurons (DeAngelis, Cumming, \& Newsome, 1998; Salzman, Britten, \& Newsome, 1990). Therefore, neurons in area V5/MT contribute directly to perceptual decisions about visual motion and depth.

Moving away from purely sensory representation, further up the visual hierarchy, some neurons in parietal cortex seem to accumulate such evidence and represent the formation of a decision. The activity of neurons in area LIP is modulated by the strength of the sensory stimulus about which a decision is made, but it also represents the direction of the behavioral response that indicates the decision that has been reached. Shadlen and Newsome (1996, 2001) recorded from neurons in LIP while monkeys carried out a two-alternative forced-choice motiondiscrimination task with random dot stimuli, for which the strength of motion in one particular direction was varied from random to $100 \%$ coherent (Figure 3). Monkeys indicated their perceptual choice about direction of motion by an eye movement to one of two possible targets. One target was always in the response field of the recorded LIP neuron. As the monkey viewed the motion stimulus, LIP neurons altered their firing rate in a way that was predictive of the direction of motion the monkey

would choose at the end of the trial. This predictive activity started early and increased during the trial while the monkey was viewing the motion stimulus, but only if the subsequent decision was into the response field of the LIP neuron. The rise was steeper for the easier, more coherent stimuli. Shadlen and Newsome $(1996,2001)$ suggested that these changes in neuronal firing during the trial show that LIP neurons accumulate the evidence for and against a specific decision. In a reaction time version of the same motion-discrimination task, Roitman and Shadlen (2002) showed subsequently that a stronger coherent motion signal was not only associated with a steeper rise in the LIP neurons' spike rate but also led to shorter reaction times for the monkey's responses. They interpreted this result to mean that the faster rising spike rate reached a particular decision threshold earlier and thus led to an earlier decision. Electrical stimulation in LIP again influenced the decision made, confirming LIP's direct contribution to the process (Hanks, Ditterich, \& Shadlen, 2006).

Thus, we have one brain site, LIP, where, at the level of single neurons, evidence for perceptual decisions may be integrated and the decision represented, and another, V5/MT, that represents perceptual evidence for decision making. To our knowledge, no experiment has yet investigated the contribution of social information in these tasks at the level of single neurons. So could these be appropriate sites for integration of social information into the decision processas the Berns et al. (2005) study suggests? Since neurons in area LIP represent the subsequent decision (if the monkey is required to make an eye-movement response), we would expect that the activity of LIP neurons is modulated by social influence whenever the decision itself is affected. But even on trials in which social influence does not change the ultimate decision, social information might alter how fast a decision is reached, and, if it does, its impact should be measurable in the slope of the rising phase of the neuronal response. Thus, with neurophysiological recordings in the awake macaque, we could gain an understanding of how and over what time course social influence affects decision processes. An elegant, technically very demanding

No Correlation

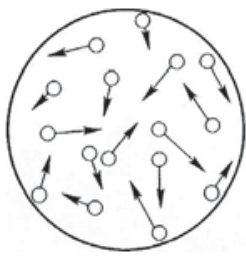

Figure 3. The random motion (or zero-coherence motion) stimulus consists of random dots, which are repositioned at a new random location on each computer frame (Newsome \& Paré, 1988). Dots appear to move in all directions with no overall motion vector (left). The larger the level of motion coherence, the greater is the proportion of dots moving unambiguously in one direction (middle and right). Figure from "The Analysis of Visual Motion: A Comparison of Neuronal and Psychophysical Performance," by K. H. Britten, M. N. Shadlen, W. T. Newsome, and J. A. Movshon, 1992, Journal of Neuroscience, 12, p. 4746. Copyright 1992 by the Society for Neuroscience. Reprinted with permission. 
study has recently shown that the neuronal activity in the parietal cortex is modulated during social interactions of 2 monkeys. Fujii, Hihara, and Iriki (2007) recorded from the parietal cortex of 2 monkeys simultaneously. When the monkeys did not interact physically, neurons were generally responding robustly to self-movements of the contralateral arm. But when monkeys were in positions in which they could both reach for food in a shared space, recorded responses changed to reflect complex interactions involving self-motion, motion of the other, as well as dominance/ subordinance of monkeys. Because of the complexity of the interactions, further studies will be needed to discriminate what causes the changes in neuronal activity and what might be their direct behavioral consequences.

Could social influence not only affect neuronal activity in LIP but also responses of sensory neurons in visual cortex? Let us return to our example of a monkey foraging for food in the trees. Faced with a variety of information about where to move next, the monkey has to integrate evidence from multiple sources to identify the best place for finding food and not just base its decision on one cue alone. Previous research has not only shown sensory signals and perceptual evidence in extrastriate visual areas, but also that neuronal signals in these areas are modulated by cognitive signals such as attention or memory effects (e.g., Bisley, Zaksas, Droll, \& Pasternak, 2004; Treue \& Maunsell, 1999). As we know from human psychophysics discussed earlier, social information can provide evidence to support a specific perceptual decision. And if Berns et al. (2005) are right and social information can affect perceptual decisions through altering what we see, we would expect to find neuronal traces relating to social information in visual cortical neurons as well.

Only new experiments can address these questions, carefully distinguishing these possibilities. Perceptual decision paradigms provide plenty of scope to study social influences in decision making in humans and, importantly, also in monkeys, at the level of single neurons. As we lay out in this section, recordings in awake behaving monkeys have already provided detailed functional models of perceptual decision making. Available models of the neuronal processes underlying perceptual decision making suggest potential brain sites where social information might be integrated into the decision process. Furthermore, electrical microstimulation in behaving animals has established causal links between neurons in these areas and decision making. To go beyond correlations between brain areas and behavior and to be able to potentially investigate the causal interactions between the activity in neurons and decision processes is another important advantage of a paradigm that can be used in nonhuman primates.

A new paradigm. In order to study social influence in perceptual decision making, we can extend the perceptual decision paradigm previously used in monkey neurophysiology to include "advice" provided by a conspecific. This could be implemented as follows: In the first phase of the trial, a macaque monkey views a stimulus - for instance, a random motion stimulus. In the second phase, the monkey watches an inset video clip of a conspecific making a decision about the direction of motion of the same stimulus (the conspecific is using a touch screen). Finally, in the third phase, the monkey views the stimulus again, and has to make a decision. If the monkey makes a correct decision, a reward is given.

Such a paradigm offers the possibility of creating a situation in which the perception of the monkey is at odds with the conspecific's decision. To illustrate, imagine a patch of dots moving unambiguously to the left, but the monkey is presented with a video clip in which the conspecific makes the choice that the dots move right. Note that this situation parallels the situation with which human participants were confronted in the Asch $(1952,1956)$ studies (with the exception that the participants were not only confronted with one other decision). But we know from Subiaul et al. (2004) that rhesus monkeys can learn from watching the behavioral choices of just one other conspecific. In such a situation, the monkey could follow either the stimulus presented or the conspecific's decision. This would allow us to compare the neural activity predicting the monkey's decision when the monkey responds correctly to the visual stimulus and on trials when it follows - incorrectly - the conspecific's choice. We could investigate directly at what level of brain processing - for instance, visual or parietal cortex - neuronal activity reflects social influence. Furthermore, we should see when social influence affects decisionrelated signals: We might see changes in baseline activity before the onset of the stimulus after the conspecific made its choice. We could also investigate how social influence modulates decision-related signals: For instance, do we see a step change in activity or more dynamic changes (i.e., a rising or falling correlate of social influence during the second stimulus presentation)? Lastly, we might be able to use electrical microstimulation at functionally identified sites to mimic or counteract the effects of social influence.

To implement such a task experimentally, various issues need to be considered; for example, what training the monkey should receive, the frequency of presented correct and incorrect choices by the conspecific, and crucially, the reward schedule. We will discuss briefly some of these issues below. Of course, some of these parameters would be decided on the basis of behavioral data that will need to be collected.

Initially, the monkeys should be trained on the perceptual task without another conspecific making a decision. When the monkey's performance has reached criterion on the visual-discrimination task, we can test the effect of social influence on behavior. At this point, psychophysical studies will have to establish the extent to which the display of the conspecific's choice influences the monkey's decision-in the absence of specific training. The perceptual decision task should be difficult - that is, near threshold - so there is an incentive for the monkey to utilize all available information. The percentage of "bad" advice should be tightly controlled; its likelihood should initially be very low, maybe $5 \%-10 \%$ of trials. Careful consideration needs to be given to the reward schedule on these trials, because the probability of reward affects monkeys' decision making (Sugrue, Corrado, \& Newsome, 2004, 2005). All of these parameters can influence subsequent behavior by the monkey. If social influence in this paradigm and setup is naturally strong, 
and a low frequency of trials with bad advice is used, one possibility would be to simply reward according to the visual stimulus. But we have to consider the possibility that a monkey might simply ignore the conspecific's choices. In this case, the monkey could be trained with high task difficulty and correct advice only, before incorrect advice is introduced in a small number of interleaved trials. Rhesus monkeys have exhibited optimal strategic behavior in several experiments when playing complex games against a computer, the strategy of which was dynamically controlled (e.g., Dorris \& Glimcher, 2004; Lee, Conroy, McGreevy, \& Barraclough, 2004). This suggests that monkeys can integrate different cues and utilize reinforcement learning algorithms to optimize behavioral responses dynamically. Thus, if necessary, to train a monkey to take into account a social cue should be reasonably straightforward. Importantly, we would argue, in the appropriate setting, this should not be necessary, because behavioral evidence presented above suggests that social perceptual learning is part of monkeys' natural repertoire (Meunier et al., 2007).

One question that can be addressed with such a paradigm is how the ambiguity of the visual stimulus affects the influence of the conspecific's choice. On a behavioral level, social psychology predicts that the monkey will rely more on conspecifics' decisions when the stimulus is ambiguous and, hence, the monkey is uncertain about which decision is correct: Informational influence should increase when the stimulus is ambiguous (e.g., Baron et al., 1996). It would be very interesting to examine the neural correlates of this phenomenon at the neuronal level.

At this point, it is important to note that we are more likely to see informational social influence than we are to be able to study normative aspects of social decision making in the nonhuman primate, especially in the controlled neurophysiological setup. If a monkey watches a video clip of a conspecific making a decision, without the conspecific being physically present, normative influence is unlikely to occur. One possible way of increasing the likelihood of normative influence would be to have a low-status monkey watch a video of a higher ranking conspecific making a decision. However, this may simultaneously increase the likelihood that informational influence is operating, since a higher ranking conspecific may be perceived as more competent. Note that this intriguing potential interaction between normative and informational social influence is not restricted to studying nonhuman primates, but also has to be carefully addressed in human decision-making experiments. For instance, conforming to a majority can be due to both the need to avoid being ostracized ("normative influence") and the belief that the majority must be right because several pairs of eyes are better than one ("informational influence").

We can pose additional questions within the framework of such a paradigm. In this context, it will also be important to take into consideration the timing of social influence: before a stimulus is viewed, during viewing, or after viewing. If information from other decision makers is provided before or during viewing of the stimulus, neuronal signals corresponding to attention to a particular perceptual decision (similar to "feature attention") or neuronal signals correlating with a particular response bias could influence decision making at the level of visual cortex (Dodd et al., 2001; Krug, 2004; Parker, Curnow, Cumming, \& Krug, 2002; Treue \& Maunsell, 1999). Control experiments (like in Berns et al., 2005) comparing responses when social influence cues a perceptual interpretation versus when an equivalent abstract cue is used could aid discrimination of such effects. If social information is provided after stimulus viewing, it seems more likely that a perceptual decision has already been reached. Here, social information might influence the decision at the levels where the decision and/or the appropriate behavioral response associated with this decision are represented (if we can distinguish between the two in the monkey). However, the visual cortex may still play a role, because it has been shown to contain memory traces for visual discrimination tasks and thus might be involved in retaining information about the stimulus or in recalling the stimulus (Bisley et al., 2004).

Another factor that needs to be closely controlled will be the role of reward. Sugrue et al. $(2004,2005)$ have shown that reward history affects choice behavior in monkeys as well as activity in LIP. It needs to be considered that the monkey might code the choice by the conspecific as a change in reward probability for a particular decision; this possibility needs to be explored when analyzing the behavioral and neuronal data. In the proposed study, not only the role of reward history of the "judge" monkey would need to be considered, but also whether there is an effect of viewing the "advisor" monkey taking rewards. Indeed, the possibility has to be considered that viewing another monkey receiving a reward - in the context of the monkey's own reward history - might have a stronger influence on decision making than does the choice the conspecific makes. Using different "advice" video clips with or without showing "reward" for the advisor monkey could explore this issue.

In summary, studying social influence on perceptual decision making in the rhesus monkey provides an experimental framework in which we can investigate the neuronal mechanism by which social influence affects decision making.

\section{Conclusions}

Decision making does not occur in a social vacuum. Whereas neuroeconomics has started to examine the brain activity in situations in which human decision makers strategically interact with others for monetary reward, other aspects of social decision making, such as the neural underpinnings of integrating the opinions of others, are only beginning to be examined (see Berns et al., 2005; GomezBeldarrain et al., 2004). In contrast, for over 50 years, social psychologists have been investigating how decision makers are influenced by others' opinions, thereby demonstrating the tremendous impact of unanimous majorities, even if the majority is obviously incorrect. This research has elucidated the factors that determine whether people conform: either because they have a need for acceptance and social approval or because they want to obtain accurate information and remove ambiguity. Moreover, research on minority influence shows that even a small number of dissenters can exert a big influence on the other group members' decision processes.

Social influence applied to perceptual decision paradigms could provide a powerful tool to research social 
decisions in general, because (1) these paradigms have been successfully used in psychophysics, fMRI, and electrophysiology; (2) they can be used with humans and monkeys; (3) the degree of difficulty of the decision can be adjusted, at least in humans, to study both informational influence as well as normative influence; and (4) we already have a good understanding of the neuronal processes that govern at least some of the perceptual decision tasks themselves. For perceptual decision making, the available data suggest that the formation of a decision takes place - at least partially — within the brain areas of the occipital and parietal cortex. At the level of single neurons, the integration of sensory evidence and other cognitive factors has been characterized. The causal importance of these sites for perceptual decision making has been underlined through electrical microstimulation. Adding to this evidence, the available neuroimaging data on social influence in perceptual decisions render areas in the occipital and parietal cortex strong contenders for integration of social influence into the decision process. In turn, neuroeconomics experiments and lesions in patients suggest that brain areas in the frontal cortex, the anterior cingulate, and the basal ganglia could be the source for the cognitive and emotional processing of the social context that then is integrated into the decision process. On the basis of reward expectations, value judgments, or emotional reactions, these circuits could provide the weighting of social influence. Studying social influence at the level of neuronal circuitry in the monkey will allow us to investigate where and how the integration of social influence into the decision process might take place.

Crucially, perceptual learning and foraging paradigms have provided evidence that monkeys can learn by watching others (e.g., Bonnie \& de Waal, 2007; Subiaul et al., 2004). By observing as well as receiving instruction from other conspecifics, an individual becomes subject to cultural influences. Although there are many aspects to social decision making that appear unique to humans, and we might ultimately apply much more complex strategies when we make decisions than do other animals, the influence of others is one facet of decision making we seem to have in common across many species. How strong this social influence can be-even for simple choices - has been shown in many social psychological experiments, to the astonishment of researchers such as Asch (1952, 1956). By combining perceptual decision making and visual instruction about the decision made by a conspecific, we can probe the fundamental processes that underlie the social influence of the opinion of others in decision making. Such experiments can provide an important stepping stone to an understanding of how we integrate and weigh multiple opinions in more complex decision tasks.

\section{AUTHOR NOTE}

K.K. is a Royal Society University Research Fellow. The collaboration between the authors was made possible by a grant from the Volkswagen Foundation, and the article was written as part of the "European Platform for Life Sciences, Mind Sciences, and the Humanities" initiative of the Volkswagen Foundation. The authors thank the members of the Platform for inspiring discussions; in particular, Philippe Tobler, Tobias
Kalenscher, Nick Shea, and Stefan Kaiser. Correspondence concerning this article should be addressed to A. Mojzisch, Institute of Psychology, Georg-August-Universität Göttingen, 37073 Göttingen, Germany (e-mail: mojzisch@psych.uni-goettingen.de).

\section{REFERENCES}

Asch, S. E. (1952). Social psychology. Englewood Cliffs, NJ: Prentice Hall.

Asch, S. E. (1956). Studies of independence and conformity: I. A minority of one against a unanimous majority. Psychological Monographs, 70(9, Whole No. 416).

Baron, R. S., Vandello, J. A., \& Brunsman, B. (1996). The forgotten variable in conformity research: Impact of task importance on social influence. Journal of Personality \& Social Psychology, 71, 915-927.

Berns, G. S., Chappelow, J., Zink, C. F., Pagnoni, G., MartinSKURSKI, M. E., \& RichaRdS, J. (2005). Neurobiological correlates of social conformity and independence during mental rotation. Biological Psychiatry, 58, 245-253.

Bisley, J. W., Zaksas, D., Droll, J. A., \& PasternaK, T. (2004). Activity of neurons in cortical area MT during a memory for motion task. Journal of Neurophysiology, 91, 286-300.

Bonaccio, S., \& Dalal, R. S. (2006). Advice taking and decisionmaking: An integrative literature review, and implications for the organizational sciences. Organizational Behavior \& Human Decision Processes, 101, 127-151.

BonNIE, K. E., \& DE WAAL, F. B. M. (2007). Copying without rewards: Socially influenced foraging decisions among brown capuchin monkeys. Animal Cognition, 10, 283-292.

Britten, K. H., Newsome, W. T., Shadlen, M. N., Celebrini, S., \& Movshon, J. A. (1996). A relationship between behavioral choice and the visual responses of neurons in macaque MT. Visual Neuroscience, 13, 87-100.

Britten, K. H., Shadlen, M. N., Newsome, W. T., \& Movshon, J. A. (1992). The analysis of visual motion: A comparison of neuronal and psychophysical performance. Journal of Neuroscience, 12, 4745 4765.

Brosnan, S. F., \& de WaAL, F. B. M. (2003). Monkeys reject unequal pay. Nature, 425, 297-299.

Cialdini, R. B., \& Goldstein, N. J. (2004). Social influence: Compliance and conformity. Annual Review of Psychology, 55, 591-621.

DeAngelis, G. C., Cumming, B. G., \& Newsome, W. T. (1998). Cortical area MT and the perception of stereoscopic depth. Nature, 394, 677-680.

De Dreu, C. K. W., \& Beersma, B. (2001). Minority dissent in organizational teams: Implications for group innovation. In C. K. W. De Dreu $\&$ N. K. De Vries (Eds.), Group consensus and minority influence: Implications for innovation (pp. 258-283). Oxford: Blackwell.

Deutsch, M., \& Gerard, H. B. (1955). A study of normative and informational influences upon individual judgment. Journal of Abnormal \& Social Psychology, 51, 629-636.

DE WAAL, F. B. M. (1998). No imitation without identification. Behavioral \& Brain Sciences, 21, 689.

Dodd, J. V., Krug, K., Cumming, B. G., \& Parker, A. J. (2001). Perceptually bistable three-dimensional figures evoke high choice probabilities in cortical area MT. Journal of Neuroscience, 21, 4809-4821.

Doms, M., \& Van Avermaet, E. (1980). Majority influence, minority influence and conversion behavior: A replication. Journal of Experimental Social Psychology, 16, 283-292.

DORRIS, M. C., \& GLIMCHER, P. W. (2004). Activity in posterior parietal cortex is correlated with the relative subjective desirability of action. Neuron, 44, 365-378.

Fehr, E., \& CAMERER, C. F. (2007). Social neuroeconomics: The neural circuitry of social preferences. Trends in Cognitive Sciences, 11, 419-427.

Fragaszy, D., \& Visalberghi, E. (2004). Socially biased learning in monkeys. Learning \& Behavior, 32, 24-35.

FuJII, N., Hihara, S., \& IrIKI, A. (2007). Dynamic social adaptation of motion-related neurons in primate parietal cortex. PLOS ONE, 2, e397.

GLIMcher, P. W. (2003). Decisions, uncertainty, and the brain: The science of neuroeconomics. Cambridge, MA: MIT Press.

Gold, J. I., \& ShadLEN, M. N. (2007). The neural basis of decision making. Annual Reviews of Neuroscience, 30, 535-574. 
Gomez-Beldarrain, M., Harries, C., Garcia-Monco, J. C., BalLus, E., \& Grafman, J. (2004). Patients with right frontal lesions are unable to assess and use advice to make predictive judgments. Journal of Cognitive Neuroscience, 16, 74-89.

Hanks, T. D., Ditterich, J., \& Shadlen, M. N. (2006). Microstimulation of macaque area LIP affects decision-making in a motion discrimination task. Nature Neuroscience, 9, 682-689.

Hastie, R., \& Kameda, T. (2005). The robust beauty of majority rules in group decisions. Psychological Review, 112, 494-508.

Heekeren, H. R., Marrett, S., \& Ungerleider, L. G. (2008). The neural systems that mediate human perceptual decision making. $\mathrm{Na}$ ture Reviews Neuroscience, 9, 467-479.

Jordan, K., Heinze, H.-J., Lutz, K., Kanowski, M., \& Jäncke, L. (2001). Cortical activations during the mental rotation of different visual objects. Neurolmage, 13, 143-152.

KerR, N. L., \& Tindale, R. S. (2004). Group performance and decision making. Annual Review of Psychology, 55, 623-656.

KNOCH, D., \& FeHr, E. (2007). Resisting the power of temptations. The right prefrontal cortex and self control. Annals of the New York Academy of Sciences, 1104, 123-134.

KRUG, K. (2004). A common neuronal code for perceptual processes in visual cortex? Comparing choice and attentional correlates in V5/MT. Philosophical Transactions of the Royal Society B, 359, 929-941.

Krug, K., Cumming, B. G., \& PArker, A. J. (2004). Comparing perceptual signals of V5/MT neurons in two binocular depth tasks. Journal of Neurophysiology, 92, 1586-1596.

Larson, J. R., Christensen, C., Авbott, A. S., \& Franz, T. M. (1996). Diagnosing groups: Charting the flow of information in medical decision-making teams. Journal of Personality \& Social Psychology, 71, 315-330.

Lee, D., Conroy, M. L., McGreevy, B. P., \& Barraclough, D. J. (2004). Reinforcement learning and decision making in monkeys during a competitive game. Cognitive Brain Research, 22, 45-58.

LeVIne, J. M., \& KaARBo, J. (2001). Minority influence in political decision-making groups. In C. K. W. De Dreu \& N. K. De Vries (Eds.), Group consensus and minority influence: Implications for innovation (pp. 229-257). London: Blackwell.

Loewenstein, G., Rick, S., \& Cohen, J. D. (2008). Neuroeconomics. Annual Reviews of Psychology, 59, 647-672.

MARTIN, R. (1998). Majority and minority influence using the afterimage paradigm: A series of attempted replications. Journal of Experimental Social Psychology, 34, 1-26.

Martin, R., \& Hewstone, M. (2003). Social influence processes of control and change: Conformity, obedience to authority, and innovation. In M. A. Hogg \& J. Cooper (Eds.), Sage handbook of social psychology (pp. 347-366). London: Sage.

Meunier, M., Monfardini, E., \& Boussaoud, D. (2007). Learning by observation in rhesus monkeys. Neurobiology of Learning \& Memory, 88, 243-248.

Mojzisch, A., Schulz-Hardt, S., Kerschreiter, R., Brodbeck, F. C., \& FrEY, D. (2008). Social validation in group decision making: Differential effects on the decisional impact of preference-consistent and preference-inconsistent information. Journal of Experimental Social Psychology, 44, 1477-1490.

Moscovici, S. (1976). Social influence and social change. London: Academic Press.

Moscovici, S. (1980). Toward a theory of conversion behavior. In L. Berkowitz (Ed.), Advances in experimental social psychology (Vol. 13, pp. 209-239). New York: Academic Press.

Moscovici, S., Lage, E., \& Naffrechoux, M. (1969). Influence of a consistent minority on the responses of a majority in a color perception task. Sociometry, 32, 365-380.

Moscovici, S., \& Personnaz, B. (1980). Studies in social influence: $\mathrm{V}$. Minority influence and conversion behavior in a perceptual task. Journal of Experimental Social Psychology, 16, 270-282.

MYERS, W. A. (1970). Observational learning in monkeys. Journal of the Experimental Analysis of Behavior, 14, 225-235.

Newsome, W. T., Britten, K. H., \& Movshon, J. A. (1989). Neuronal correlates of a perceptual decision. Nature, 341, 52-54.
Newsome, W. T., \& Paré, E. B. (1988). A selective impairment of motion perception following lesions of the middle temporal visual area (MT). Journal of Neuroscience, 8, 2201-2211.

Parker, A. J., Curnow, T. L., Cumming, B. G., \& Krug, K. (2002). Temporal dynamics of choice-related neuronal firing in macaque V5/ MT. Society for Neuroscience Abstracts, 28, 121.10.

RoITMAN, J. D., \& SHADLEN, M. N. (2002). Response of neurons in posterior parietal cortex (area LIP) during a combined reaction-time directiondiscrimination task. Journal of Neuroscience, 22, 9475-9489.

Rudebeck, P. H., Buckley, M. J., Walton, M. E., \& Rushworth, M. F. (2006). A role for the macaque anterior cingulate gyrus in social valuation. Science, 313, 1310-1312.

Rushworth, M. F., Behrens, T. E., Rudebeck, P. H., \& WalTON, M. E. (2007). Contrasting roles for cingulate and orbitofrontal cortex in decisions and social behaviour. Trends in Cognitive Sciences, 11, 168-176.

Salzman, C. D., Britten, K. H., \& Newsome, W. T. (1990). Cortical microstimulation influences perceptual judgments of motion direction. Nature, 346, 174-177.

SAnfey, A. G. (2007). Social decision-making: Insights from game theory and neuroscience. Science, 318, 598-602.

Schulz-Hardt, S., Brodbeck, F. C., Mojzisch, A., Kerschreiter, R., \& FREY, D. (2006). Group decision making in hidden profile situations: Dissent as a facilitator for decision quality. Journal of Personality \& Social Psychology, 91, 1080-1093.

Shadlen, M. N., \& Newsome, W. T. (1996). Motion perception: Seeing and deciding. Proceedings of the National Academy of Sciences, 93, 628-633.

Shadlen, M. N., \& Newsome, W. T. (2001). Neural basis of a perceptual decision in the parietal cortex (area LIP) of the rhesus monkey. Journal of Neurophysiology, 86, 1916-1936.

Shepard, R. N., \& Metzler, J. (1971). Mental rotation of threedimensional objects. Science, 171, 701-703.

SHERIF, M. (1936). The psychology of social norms. New York: Harper \& Row.

Somerville, L. H., Heatherton, T. F., \& Kelley, W. M. (2006). Anterior cingulate cortex responds differentially to expectancy violation and social feedback. Nature Neuroscience, 9, 1007-1008.

Sorrentino, R. M., King, G., \& Leo, G. (1980). The influence of the minority on perception: A note on a possible alternative explanation. Journal of Experimental Social Psychology, 16, 293-301.

Subiaul, F., Cantlon, J. F., Holloway, R. L., \& Terrace, H. S. (2004). Cognitive imitation in rhesus macaques. Science, 305, 407-410.

Sugrue, L. P., Corrado, G. S., \& Newsome, W. T. (2004). Matching behavior and the encoding of value in parietal cortex. Science, $\mathbf{3 0 4}$, 1782-1787.

Sugrue, L. P., Corrado, G. S., \& Newsome, W. T. (2005). Choosing the greater of two goods: Neural currencies for valuation and decision making. Nature Reviews Neuroscience, 6, 363-375.

Treue, S., \& MAunsell, J. H. (1999). Effects of attention on the processing of motion in macaque middle temporal and medial superior temporal visual cortical areas. Journal of Neuroscience, 19, 7591-7602.

UKa, T., \& DeAngelis, G. C. (2004). Contribution of area MT to stereoscopic depth perception: Choice-related response modulations reflect task strategy. Neuron, 42, 297-310.

Visalberghi, E., \& Fragaszy, D. M. (2002). Do monkeys ape? Ten years after. In K. Dautenhahn \& C. Nehaniv (Eds.), Imitation in animals and artifacts (pp. 471-499). Cambridge, MA: MIT Press.

von Neumann, J., \& Morgenstern, O. (1944). Theory of games and economic behavior. New York: Wiley.

Yaniv, I. (2004). The benefit of additional opinions. Current Directions in Psychological Science, 13, 75-78.

Zuberbühler, K., Gygax, L., Harley, N., \& Kummer, H. (1996). Stimulus enhancement and spread of a spontaneous tool use in a colony of long-tailed macaques. Primates, 37, 1-12.

(Manuscript received March 1, 2008; revision accepted for publication July 31,2008 .) 